\title{
The Noether-Fano inequalities for codimension one singular holomorphic foliations
}

\author{
Luís Gustavo Mendes
}

Received: date / Accepted: date

\begin{abstract}
The idea of the proof of the classical Noether-Fano inequalities can be adapted to the domain of codimension one singular holomorphic foliations of the projective space. We obtained criteria for proving that the degree of a foliation on the plane is minimal in the birational class of the foliation and for the non-existence of birational symmetries of generic foliations (except automorphisms). Moreover, we give several examples of birational symmetries of special foliations illustrating our results.
\end{abstract}

Keywords Holomorphic foliation · Noether-Fano method

Mathematics Subject Classification (2000) Primary 37F75 · Secondary 14E07

\section{Introduction}

The classical Noether-Fano inequalities give information on the multiplicities of singularities of the generic element of a homaloidal system, in terms of the algebraic degree of the associated birational map (Section 4.1.2 gives an example). They are fundamental in the Sarkisov's program of factorization of birational transformations and are used to prove that birational transformations of some Fano varieties are in fact isomorphisms.

We have adapted the general idea of the proof (cf. [9]) to codimension one singular holomorphic foliations. We call the attention to the fact that even in the case when a foliation is a pencil of algebraic curves, the study that we developed cannot be reduced to the classical one. The reason is that there is no general relation between the data of the curves (degrees, singularities) and the data of the foliation, as shown by the works on the Poincaré problem for pencils (see e.g. [6] and references therein).

$\overline{\text { Partially supported by CNPq }}$ - Brazil

L. G. Mendes

Departamento de Matemática, Universidade Federal do Rio Grande do Sul - UFRGS Av. Bento Gonçalves, 9500, Porto Alegre - RS - BRASIL. cep 91509-900

E-mail: mendes@mat.ufrgs.br 
1.1 General result

Along all the paper we deal with complex codimension one holomorphic foliations, whose singular sets have codimension greater or equal to 2 (foliations, for brevity). For a foliation $\mathcal{F}^{\prime}$ of a complex algebraic variety $N$ and for a birational transformation $\chi: M-\rightarrow N$, it is well-defined the strict transform of $\mathcal{F}^{\prime}$ in $M$, denoted $\mathcal{F}=\chi_{*}^{-1}\left(\mathcal{F}^{\prime}\right)$. Namely, it is the unique foliation which extends $\left(\chi_{\mid U}\right)^{*}\left(\mathcal{F}_{\mid \chi(U)}^{\prime}\right)$, for $U \subset M$ the Zariski open set where $\chi$ restricts as an isomorphism.

Let us consider two notions on foliations which will be used along all this work. Firstly, as usual the degree of a foliation $\mathcal{F}$ of the projective space is defined as the sum of orders of contact of a straight line with the codimension one leaves of $\mathcal{F}$. Secondly, a natural notion of multiplicity:

Definition 1 Let $\sigma=\sigma_{\Sigma}$ be a blowing up of a smooth center $\Sigma$, with exceptional divisor $E_{\Sigma}=\sigma^{-1}(\Sigma)$. If $\mathcal{F}=\sigma_{*}^{-1}\left(\mathcal{F}^{\prime}\right)$, there is an isomorphism:

$$
N_{\mathcal{F}}=\sigma_{\Sigma}^{*}\left(N_{\mathcal{F}^{\prime}}\right) \otimes\left(-\alpha E_{\Sigma}\right)
$$

for an integer $\alpha \geq 0\left(\alpha \geq 1\right.$ if $\left.\Sigma \subset \operatorname{sing}\left(\mathcal{F}^{\prime}\right)\right)$. By definition $l\left(\Sigma, \mathcal{F}^{\prime}\right):=\alpha$.

Section 2.1 is dedicated to this theorem-definition. We want to emphasize that even in dimension two, there is fundamental difference between the behavior of $l(p, \mathcal{F})$ and the behavior of the usual multiplicity of a curve at a point; in fact, $l(p, \mathcal{F})$ may increase after blowing ups, that is, it may happens that $l\left(q, \sigma_{*}^{-1}(\mathcal{F})\right)>l(p, \mathcal{F})$, where $q \in \sigma^{-1}(p)$. Section 2.1.1 exemplifies this fact with dicritical and nilpotent singularities of foliations. This makes difficult the adaptation of all Sarkisov's program to foliations.

In higher dimension, birational transformations $\chi: \mathbf{C} P^{N}-\rightarrow \mathbf{C} P^{N}$ can be factorized in a weak sense, by Hironaka's elimination of indeterminations $\operatorname{Ind}(\chi)$. There is a morphism $\sigma: M \rightarrow \mathbf{C} P^{N}$, where $\sigma:=\sigma_{1} \circ \ldots \circ \sigma_{k}$ is a finite sequence of blowing ups $\sigma_{i}$ along smooth centers $\Sigma_{j} \subset \operatorname{Ind}(\chi), \operatorname{codim}\left(\Sigma_{j}\right) \geq 2$ and there is morphism $f: M \rightarrow \mathbf{C} P^{N}$ such that

$$
\chi=f \circ \sigma^{-1} .
$$

In higher dimension, we say that a smooth variety $\Sigma \subset \operatorname{Ind}(\chi)$ has divisorial birational image by $\chi$ factorized as in (2), if the exceptional divisor $E_{\Sigma}$ introduced at some step of the sequence $\sigma$ has a divisorial image by $f$. The classical cubo-cubic Cremona transformation of $\mathbf{C} P^{3}$ has a smooth connected curve of indetermination with divisorial birational image, see Section 4.2.1. Differently, Section 4.2.2 recalls that some centers contained in the indetermination set of the standard cubic Cremona transformation of $\mathbf{C} P^{3}$ have no divisorial birational images. Their distinct effect on foliations is described in Section 4.2. With these notations, we can state:

Theorem 1 Let $\chi: \mathbf{C} P^{N}-\rightarrow \mathbf{C} P^{N}, N \geq 2$, be a birational transformation having a factorization $\chi=f \circ \sigma^{-1}$ as in (2). Let $\mathcal{F}$ and $\mathcal{F}^{\prime}$ be foliations of $\mathbf{C} P^{N}$ with $\mathcal{F}=$ $\chi_{*}^{-1}\left(\mathcal{F}^{\prime}\right)$.

i) Suppose that all $\Sigma_{j} \subset$ Ind $(\chi)$ having divisorial birational image by $\chi$ verify:

$$
\begin{gathered}
l\left(\Sigma_{1}, \mathcal{F}\right) \leq\left(q_{1}-1\right) \cdot \frac{d(\mathcal{F})+2}{N+1} \quad \text { and } \\
l\left(\Sigma_{j},\left(\sigma_{1} \circ \ldots \circ \sigma_{j-1}\right)_{*}^{-1}(\mathcal{F})\right) \leq\left(q_{j}-1\right) \cdot \frac{d(\mathcal{F})+2}{N+1}, \quad q_{j}:=\operatorname{codim}\left(\Sigma_{j}\right), \forall j \geq 1 .
\end{gathered}
$$


Then $d(\mathcal{F}) \leq d\left(\mathcal{F}^{\prime}\right)$.

ii): Supposing i), then $d(\mathcal{F})=d\left(\mathcal{F}^{\prime}\right)$ is equivalent to equalities in (3) and (4).

Several examples for this result are given in Sections 4.1.2 and 4.2.3.

We remark that the classical Noether-Fano inequalities give more information on the centers $\Sigma_{j}$ than Theorem 1 (cf. Examples 9.1.4 of [7]). But this extra information depends on Nefness properties which are lost, in general, in the adaptation to foliations.

\subsection{Results in dimension two}

When we particularize our result to dimension two, any indetermination point has divisorial birational image, i.e. a curve. Also, in the factorization (2), $f$ is a sequence of blowing ups of points.

Moreover, after finitely many blowing-ups, the strict transform of any foliation has at most reduced singularities (e.g. [1]). For reduced singularities the multiplicity defined in (1) is at most one and does not increase under extra blowing-ups.

Let us introduce the notion of birational degree of $\mathcal{F}$, as the minimum degree of foliations of a projective space which can be birationally transformed into $\mathcal{F}$.

We shall adopt a simplifying notation (detailed in Section 2.3), where $l\left(p, \sigma_{*}^{-1}(\mathcal{F})\right.$ ) simplifies to $l(p, \mathcal{F})$. An immediate consequence of Theorem 1-i) is:

Corollary 1 Let $\mathcal{F}$ in $\mathbf{C} P^{2}$ with $l(p, \mathcal{F}) \leq \frac{d(\mathcal{F})+2}{3}, \forall p \in \operatorname{sing}(\mathcal{F})$ as well as for all singularities of foliations of each step of a reduction of singularities of $\mathcal{F}$.

Then $d(\mathcal{F})$ is the birational degree.

Corollary 2 Let $\mathcal{F}$ in $\mathbf{C} P^{2}$ with $d(\mathcal{F}) \geq 2$ and $l(p, \mathcal{F})=1, \forall p \in \operatorname{sing}(\mathcal{F})$ as well as for all singularities of foliations of each step of a reduction of singularities of $\mathcal{F}$. Suppose there are a birational map $\chi: \mathbf{C} P^{2}-\rightarrow \mathbf{C} P^{2}$ and a foliation $\mathcal{F}^{\prime}$ with $\mathcal{F}=\chi_{*}^{-1}\left(\mathcal{F}^{\prime}\right)$ and that $d(\mathcal{F})=d\left(\mathcal{F}^{\prime}\right)$.

Then in fact $\chi$ is an isomorphism of $\mathbf{C} P^{2}$.

In particular, if $\mathcal{F}$ has a birational symmetry $\chi_{*}^{-1}(\mathcal{F})=\mathcal{F}$ of a generic foliation, Corollary 2 asserts that it is an automorphism. This is a weaker version of a result of [2], [16] and [3], asserting that the group of birational symmetries of a reduced foliation with maximal foliated Kodaira dimension $\kappa=2$ (cf. [12], [1], [11]) coincides with its group of automorphisms and is a finite group.

At last, an application to pencils of curves. In his classical lectures on pencils ([18]), H. Poincaré obtained, in particular cases, bounds on the geometrical genus $g(C)$ of the generic curve of a pencil $\mathcal{F}$ in terms of the data of the foliation. For instance, we can deduce from his formulae that if at the base-points of $\mathcal{F}$ (and their infinitely near points) $l(p, \mathcal{F}) \leq \frac{d(\mathcal{F})+2}{3}$, then $g(C) \geq 1$. The particular case when the base-points of $\mathcal{F}$ are of radial type $x d y-y d x+$ h.o.t (i.e. $l(p, \mathcal{F}) \leq 2$ ) and $d(\mathcal{F}) \geq 4$ was considered by him in detail. With hypotheses not only on base-points but on all the singularities we easily prove:

Corollary 3 If $l(p, \mathcal{F}) \leq \frac{d(\mathcal{F})+2}{3}$ for all singular points of a reduction of singularities of a pencil of curves $\mathcal{F}$, then $g(C) \geq 1$. In particular, generic curves $C$ of pencils with $d(\mathcal{F}) \geq 4$ have $g(C) \geq 1$, provided that each singularity of the pencil is of type $\lambda x d y-y d x+$ h.o.t, with $\lambda=\lambda(p) \neq 0$. 
The third example of Section 4.1.2 gives the birational degrees of pencils of curves with $g(C)=1$, for which $l(p, \mathcal{F})=\frac{d(\mathcal{F})+2}{3}$ at the base-points.

\section{Background for the proofs}

2.1 Remarks on $l(\Sigma, \mathcal{F})$

The isomorphism in (1) can be seen as follows: take a rational 1-form $\Omega$ with zeroes of codimension at least two inducing $\mathcal{F}^{\prime}$ and consider the divisor of zeroes of its pullback $\left(\sigma_{\Sigma}^{*}(\Omega)\right)_{0}=\alpha E_{\Sigma}, \quad \alpha \geq 0$. This means that $\sigma_{\Sigma}^{*}\left(N_{\mathcal{F}^{\prime}}^{*}\right)=N_{\mathcal{F}}^{*} \otimes\left(-\alpha E_{\Sigma}\right)$, where $N_{\mathcal{F}^{\prime}}^{*}$ is the co-normal line bundle. Dualizing it we get (1).

If $e$ is a generic line of the ruling of $E_{\Sigma}$ then:

$$
N_{\mathcal{F}} \cdot e=\left[\sigma_{\Sigma}^{*}\left(N_{\mathcal{F}^{\prime}}\right) \otimes \mathcal{O}\left(-l\left(\Sigma, \mathcal{F}^{\prime}\right) \cdot E_{\Sigma}\right)\right] \cdot e=l\left(\Sigma, \mathcal{F}^{\prime}\right) .
$$

When $\operatorname{dim}_{\mathbf{C}}(\Sigma) \geq 1$ this informs us that $l(\Sigma, \mathcal{F})$ can be computed as

$$
l(\Sigma, \mathcal{F})=l(p, \Sigma, \mathcal{F}):=\operatorname{ord}_{E}\left(\sigma_{\Sigma_{p}}^{*}\left(\eta_{p}\right)\right)_{0},
$$

where $\eta_{p}$ is a local holomorphic 1 -form representing $\mathcal{F}$ around $p, \Sigma_{p}:=(\Sigma, p)$, and $p$ belongs to the open dense set $V \subset \Sigma$ where $l(p, \Sigma, \mathcal{F})$ has minimal value. By other side, $l(p, \Sigma, \mathcal{F})$ does not depend on the local 1 -form $\eta_{p}$, just on $\mathcal{F}$, because another local 1 -form defining $\mathcal{F}$ is of type $g \cdot \eta$, for $g \in \mathcal{O}^{*}$.

\subsection{1 $l(p, \mathcal{F})$ can increase after blowing ups}

A useful remark on surfaces is that, when the exceptional curve of the blowing up of $p$ is not invariant by $\sigma_{*}^{-1}(\mathcal{F})$, then $l(p, \mathcal{F})=m(p, \mathcal{F})+1$, where $m(p, \mathcal{F})$ is the order of the first non-zero jet of a local 1-form representing $\mathcal{F}$; otherwise, $l(p, \mathcal{F})=m(p, \mathcal{F})$.

Let us exemplify this, starting with $\eta_{1}:=2 x d y-y d x=0$. We blow up with $\sigma(t, y)=(t y, y)=(x, y)$, obtaining $\sigma^{*}\left(\eta_{1}\right)=y \cdot(t d y-y d t)$, where $y=0$ is the exceptional divisor, so $l\left(p, \mathcal{F}_{\eta_{1}}\right)=1$. Re-start now with $\eta_{2}:=t d y-y d t$ and blow up it with $\sigma(t, u)=(t, t u)=(t, y)$, obtaining $\sigma^{*}\left(\eta_{2}\right)=t^{2} \cdot d u$; so $l\left(p, \mathcal{F}_{\eta_{2}}\right)=2$.

For another example, start with a singularity $\eta_{1}:=(y+x y) d y+\left(y^{2}-x^{3}\right) d x=0$ with nilpotent linear part. Let $\sigma(x, t)=(x, x t)=(x, y)$ and $\sigma^{*}\left(\eta_{1}\right)=x \cdot\left[\left(t^{2}+2 x t^{2}-x^{2}\right) d x+\right.$ $\left.\left(t x+x^{2} t\right) d t\right]$, that is $l\left(p, \mathcal{F}_{\eta_{1}}\right)=1$. Again blow up $\eta_{2}:=\left(t^{2}+2 x t^{2}-x^{2}\right) d x+\left(t x+x^{2} t\right) d t$ with $\sigma(x, u)=(x, x u)=(x, t)$, obtaining $l\left(p, \eta_{2}\right)=2$, because $\sigma^{*}\left(\eta_{2}\right)=x^{2} \cdot\left[\left(u^{2}(2+\right.\right.$ $\left.3 x)-1) d x+x\left(1+u x^{2}\right) d u\right]$.

\subsection{Degree of a foliation and the normal line bundle}

Let $\mathcal{F}$ be foliation of $\mathbf{C} P^{N}$. Take $\Omega$ a regular integrable section of $\Omega_{\mathbf{C} P^{N}}^{1} \otimes N_{\mathcal{F}}$ with zero set of codimension greater or equal to 2 , inducing the foliation $\mathcal{F}$, where $\Omega_{\mathbf{C} P^{N}}^{1}$ is the sheaf of 1 -forms and $N_{\mathcal{F}}$ is the normal line bundle.

Let $\phi: L \rightarrow \mathbf{C} P^{N}$ be the inclusion of a generic straight line $L$ and consider the restriction $\phi^{*}(\Omega)$, with divisor of zeroes $\left(\phi^{*}(\Omega)\right)_{0}$. By definition of degree of $\mathcal{F}$, $d(\mathcal{F})=\operatorname{deg}\left(\phi^{*}(\Omega)\right)_{0}$. Since $\phi^{*}(\omega)$ is a regular section of $\Omega_{L}^{1} \otimes\left(N_{\mathcal{F}}\right)_{\mid L}$, then

$$
d(\mathcal{F})=\operatorname{deg}\left(\Omega_{L}^{1}\right)+N_{\mathcal{F}} \cdot L=-2+N_{\mathcal{F}} \cdot L
$$


and it follows:

$$
N_{\mathcal{F}}=\mathcal{O}(d(\mathcal{F})+2)
$$

2.3 Simplifying notations used in the proofs

In general we deal with a finite sequence of blowing ups of smooth centers. A first blowing up of $\Sigma_{1}$ by $\sigma_{1}:=\sigma_{\Sigma_{1}}$ produces the strict transform $\sigma_{1}^{-1}{ }_{*}\left(\mathcal{F}^{\prime}\right)$; a second blowing up $\sigma_{2}:=\sigma_{\Sigma_{2}}$ produces from it a new strict transform $\left.\sigma_{2}^{-1}{ }_{*}\left(\sigma_{1}^{-1}(\mathcal{F})\right)\right)=$ $\left(\sigma_{1} \circ \sigma_{2}\right)_{*}^{-1}\left(\mathcal{F}^{\prime}\right)$ and so on. From (1), we obtain isomorphisms:

$$
\begin{gathered}
N_{\left(\sigma_{1} \circ \sigma_{2}\right)^{-1}\left(\mathcal{F}^{\prime}\right)}=\sigma_{2}^{*}\left(N_{\sigma_{1}^{-1}\left(\mathcal{F}^{\prime}\right)}\right) \otimes \mathcal{O}\left(-l\left(\Sigma_{2}, \sigma_{1_{*}}^{-1}\left(\mathcal{F}^{\prime}\right)\right) \cdot E_{\Sigma_{2}}\right)= \\
=\left(\sigma_{2} \circ \sigma_{1}\right)^{*}\left(N_{\mathcal{F}^{\prime}}\right) \otimes \sigma_{2}^{*} \mathcal{O}\left(-l\left(\Sigma_{1}, \mathcal{F}^{\prime}\right) \cdot E_{\Sigma_{1}}\right) \otimes \mathcal{O}\left(-l\left(\Sigma_{2}, \sigma_{1_{*}}^{-1}\left(\mathcal{F}^{\prime}\right)\right) \cdot E_{\Sigma_{2}}\right) .
\end{gathered}
$$

Such notation becomes cumbersome, so we shall adopt the following simplifying notation:

$$
N_{\left(\sigma_{1} \circ \sigma_{2}\right)^{-1}{ }_{*}\left(\mathcal{F}^{\prime}\right)}=\left(\sigma_{1} \circ \sigma_{2}\right)^{*}\left(N_{\mathcal{F}^{\prime}}\right) \otimes \mathcal{O}\left(-l\left(\Sigma_{1}, \mathcal{F}^{\prime}\right) \cdot E_{\Sigma_{1}}\right) \otimes \mathcal{O}\left(-l\left(\Sigma_{2}, \mathcal{F}^{\prime}\right) \cdot E_{\Sigma_{2}}\right) .
$$

For a sequence of blowing ups $\sigma=\sigma_{1} \circ \ldots \circ \sigma_{k}$ at centers $\Sigma_{j}, j=1, \ldots k$, starting with $\mathcal{F}^{\prime}$ and arriving at $\sigma_{*}^{-1}(\mathcal{F})$ with adopt in the proofs the simplifying notation:

$$
N_{\sigma_{*}^{-1}\left(\mathcal{F}^{\prime}\right)}=\sigma^{*}\left(N_{\mathcal{F}^{\prime}}\right) \otimes \mathcal{O}\left(-\sum_{j=1}^{k} l\left(\Sigma_{j}, \mathcal{F}^{\prime}\right) E_{\Sigma_{j}}\right) .
$$

At last, there is a well-known isomorphism $K_{\hat{M}}=\sigma^{*}\left(K_{M}\right) \otimes \mathcal{O}\left((q-1) E_{\Sigma}\right)$ relating the canonical bundles of a $N$-dimensional variety $M$ and of the blown up variety $\hat{M}$ (along a codimension $q$ smooth subvariety $\Sigma \subset M$ ), which can be seen by considering the zero divisor of the pullback by $\sigma_{\Sigma}$ of a local holomorphic $N$-form. Again, for a sequence of blowing ups at centers $\Sigma_{j}$ we adopt a simplifying notation:

$$
K_{\hat{M}}=\sigma^{*}\left(K_{M}\right) \otimes \mathcal{O}\left(\sum_{j=1}^{k}\left(q_{j}-1\right) E_{\Sigma_{j}}\right)
$$

\section{Proofs}

3.1 Proof of Theorem 1:

Let $\mathcal{F}^{\prime}$ in $\mathbf{C} P^{N}$ and $\chi: \mathbf{C} P^{N}-\rightarrow \mathbf{C} P^{N}$ such that $\mathcal{F}=\chi_{*}^{-1}(\mathcal{F})$. Let $\chi=f \circ \sigma^{-1}$ be a factorization as in (2), with $\sigma: M \rightarrow \mathbf{C} P^{N}, f: M \rightarrow \mathbf{C} P^{N}$. In $M$ there is a foliation $\mathcal{G}$ such that:

$$
\mathcal{G}=\sigma^{-1}{ }_{*}(\mathcal{F}) \text { and } \mathcal{G}=f^{-1}{ }_{*}\left(\mathcal{F}^{\prime}\right),
$$

We keep the simplifying notations (8) and (9), which we write in divisorial form:

$$
N_{\mathcal{G}}=\sigma^{*}\left(N_{\mathcal{F}}\right)-\sum_{j} l\left(\Sigma_{j}, \mathcal{F}\right) E_{\Sigma_{j}} \quad \text { and } \quad K_{M}:=\sigma^{*}\left(K_{\mathbf{C} P^{N}}\right)+\sum_{j}\left(q_{j}-1\right) \cdot E_{\Sigma_{j}} .
$$


By other side:

$$
N_{\mathcal{G}}=f^{*}\left(N_{\mathcal{F}^{\prime}}\right)+G \quad \text { and } \quad K_{M}=f^{*}\left(K_{\mathbf{C} P^{N}}\right)+G^{\prime}
$$

where the supports of the divisors $G$ and $G^{\prime}$ are contained in the support of the exceptional divisor of $f$. Consider now the divisor with rational coefficients:

$$
K_{M}+\frac{N+1}{d(\mathcal{F})+2} \cdot N_{\mathcal{G}}
$$

By one side, (10) is isomorphic to:

$$
\sigma^{*}\left(K_{\mathbf{C} P^{N}}+\frac{N+1}{d(\mathcal{F})+2} N_{\mathcal{F}}\right)+\sum_{j}\left[q_{j}-1-\frac{N+1}{d(\mathcal{F})+2} \cdot l\left(\Sigma_{j}, \mathcal{F}\right)\right] E_{\Sigma_{j}},
$$

and by another side (10) is isomorphic to:

$$
f^{*}\left(K_{\mathbf{C} P^{N}}+\frac{N+1}{d(\mathcal{F})+2} N_{\mathcal{F}^{\prime}}\right)+G^{\prime}+\frac{N+1}{d(\mathcal{F})+2} \cdot G .
$$

Now take a generic straight line $r \subset \mathbf{C} P^{N}$ and consider its total transform $f^{*}(r) \subset M$. Thanks to the Projection Formula, we get:

$$
\begin{aligned}
& f^{*}(r) \cdot G=r \cdot f_{*}(G)=0, \\
& f^{*}(r) \cdot G^{\prime}=r \cdot f_{*}\left(G^{\prime}\right)=0 .
\end{aligned}
$$

Therefore intersecting $f^{*}(r)$ with (12), using the isomorphism (7) applied to $\mathcal{F}^{\prime}$ and the fact that $K_{\mathbf{C P}^{N}}=-(N+1) H$, where $H$ is a hyperplane, we get:

$$
f^{*}(r) \cdot f^{*}\left(K_{\mathbf{C} P^{N}}+\frac{N+1}{d(\mathcal{F})+2} N_{\mathcal{F}^{\prime}}\right)=-(N+1)+\frac{N+1}{d(\mathcal{F})+2}\left(d\left(\mathcal{F}^{\prime}\right)+2\right),
$$

But we can intersect $f^{*}(r)$ with the divisor in (11), which is isomorphic to (12):

$$
f^{*}(r) \cdot\left\{\sigma^{*}(0)+\sum_{j}\left[q_{j}-1-\frac{N+1}{d(\mathcal{F})+2} \cdot l\left(\Sigma_{j}, \mathcal{F}\right)\right] E_{\Sigma_{j}}\right\}
$$

and putting together these facts we conclude that:

$$
-(N+1)+\frac{N+1}{d(\mathcal{F})+2}\left(d\left(\mathcal{F}^{\prime}\right)+2\right)=\sum_{j}\left[q_{j}-1-\frac{N+1}{d(\mathcal{F})+2} \cdot l\left(\Sigma_{j}, \mathcal{F}\right)\right] f^{*}(r) \cdot E_{\Sigma_{j}} .
$$

By the Projection Formula, $f^{*}(r) \cdot E_{\Sigma_{j}}=r \cdot f_{*}\left(E_{\Sigma_{j}}\right) \geq 0$ and this number is positive if and only if $\Sigma_{j} \subset \operatorname{Ind}(\chi)$ has divisorial birational image.

By hypothesis for $\Sigma_{j} \in \operatorname{Ind}(\chi)$ with divisorial image:

$$
l\left(\Sigma_{j}, \mathcal{F}\right) \leq\left(q_{j}-1\right) \cdot \frac{d(\mathcal{F})+2}{N+1} \Leftrightarrow q_{j}-1-\frac{N+1}{d(\mathcal{F})+2} l\left(\Sigma_{j}, \mathcal{F}\right) \geq 0 .
$$

Therefore (15) gives, as asserted in i):

$$
-(N+1)+\frac{N+1}{d(\mathcal{F})+2}\left(d\left(\mathcal{F}^{\prime}\right)+2\right) \geq 0 \quad \Leftrightarrow \quad d(\mathcal{F}) \leq d\left(\mathcal{F}^{\prime}\right) .
$$


For the part ii), suppose additionally that $d(\mathcal{F})=d\left(\mathcal{F}^{\prime}\right)$; we obtain from (15):

$$
0=\sum_{j}\left[q_{j}-1-\frac{N+1}{d(\mathcal{F})+2} \cdot l\left(\Sigma_{j}, \mathcal{F}\right)\right] f^{*}(r) \cdot E_{\Sigma_{j}} .
$$

But (16) holds for all $\Sigma_{j} \subset \operatorname{Ind}(\chi)$ with $f^{*}(r) \cdot E_{\Sigma_{j}}>0$. Then (17) implies for all these $\Sigma_{j}$ :

$$
q_{j}-1-\frac{N+1}{d(\mathcal{F})+2} \cdot l\left(\Sigma_{j}, \mathcal{F}\right)=0 .
$$

Reciprocally, supposing additionally (18) for all centers with divisorial image, then (15) gives $d(\mathcal{F})=d\left(\mathcal{F}^{\prime}\right)$.

\subsection{Proofs of Corollaries}

The hypotheses of Corollary 2 imply: $l(p, \mathcal{F}) \leq 1<\frac{4}{3} \leq \frac{d(\mathcal{F})+2}{3}, \quad \forall p \in \operatorname{Ind}(\chi)$, which are the conditions of Theorem 1-i). If $d(\mathcal{F})=d\left(\mathcal{F}^{\prime}\right)$, then Theorem 1-ii) implies $l(p, \mathcal{F})=\frac{d(\mathcal{F})+2}{3}, \forall p \in \operatorname{Ind}(\chi)$. The conclusion is that the set $\operatorname{Ind}(\chi)$ must be empty. Since $\mathbf{C} P^{2}$ is a minimal surface, $\chi$ must be an isomorphism, as desired.

For the proof of Corollary 3 , observe that $d(\mathcal{F})$ is the birational degree of $\mathcal{F}$, thanks to Corollary 1 . By other side, at a base-point of a pencil or at some infinitely near point, $l(p, \mathcal{F}) \geq 2$ (cf. Section 2.1.1). Therefore $d(\mathcal{F}) \geq 4$. By absurd, suppose that $\mathcal{F}$ is a pencil of rational curves, i.e. $g(C)=0$. Then it is birationally equivalent to the pencil of straight lines, whose degree as foliation is zero: a contradiction.

For the particular case, note that the blowing ups of singularities of type $\omega=$ $\lambda x d y-y d x+$ h.o.t produce points with $l(q, \mathcal{F}) \leq 2$. If $d(\mathcal{F}) \geq 4$, then $l(p, \mathcal{F}) \leq \frac{d(\mathcal{F})+2}{3}$.

\section{Cremona maps, their effect on foliations and examples}

The degree as foliation of a pencil of hypersurfaces of degree $k$ is given by Darboux formula. For $V_{i}$ the non-reduced components with multiplicity $\mu_{i}$ and support $\left|V_{i}\right|$ :

$$
d(\mathcal{F})=2 k-2-\sum_{i} \operatorname{deg}\left(\left|V_{i}\right|\right) \cdot\left(\mu_{i}-1\right)
$$

From this we can compute, in some cases, the degree as foliations of the strict transforms of pencils. But Darboux formula is useless for codimension one foliations in general. For this reason, Propositions 1, 2 and 3 (below) are useful.

\subsection{Dimension two}

\subsubsection{Standard quadratic map}

Any Cremona map of the plane is a composition of standard quadratic maps and linear transformations. Therefore it is useful the following remark: 
Proposition 1 Let $\mathcal{F}$ be a foliation of $\mathbf{C} P^{2}$ and $Q$ be the standard quadratic transformation, with Ind $(Q)$ given by three non-colinear points $p_{1}, p_{2}, p_{3}$ in the plane. Denote $\operatorname{Ind}\left(Q^{-1}\right)=\left\{p_{1}^{\prime}, p_{2}^{\prime}, p_{3}^{\prime}\right\}$. Then

i): $d\left(Q_{*}(\mathcal{F})\right)=2 \cdot d(\mathcal{F})+2-\sum_{i=1}^{3} l\left(p_{i}, \mathcal{F}\right)$ and

ii): $l\left(p_{i}^{\prime}, Q_{*}(\mathcal{F})\right)=d(\mathcal{F})+2-l\left(p_{j}, \mathcal{F}\right)-l\left(p_{k}, \mathcal{F}\right)$, where $i, j, k \in\{1,2,3\}$ are distinct.

\section{Proof}

If $\sigma$ denotes the composition of three blowing ups at $p_{1}, p_{2}, p_{3}$, the $(-1)$-curves $\overline{r_{i j}}=\sigma^{*}\left(r_{i j}\right)-E_{i}-E_{j}$, for $r_{i j}:=p_{i} p_{j}$, are the exceptional curves of $f$ in $Q=f \circ \sigma^{-1}$. By $(6)$ in Section $2.2, d\left(Q_{*}(\mathcal{F})\right)=-2+N_{Q_{*}}(\mathcal{F}) \cdot L$, where $L$ is a straight line. Recall that $L=f_{*}\left(\sigma_{*}^{-1}(S)\right)$, where $S$ is a smooth conic passing by $p_{1}, p_{2}, p_{3}$ (i.e. belonging to the homaloidal system of $Q$ ). Then $N_{Q_{*}(\mathcal{F})} \cdot L$ can be computed as:

$$
N_{Q_{*}(\mathcal{F})} \cdot L=\left[\sigma^{*}\left(N_{\mathcal{F}}\right)-\sum_{i=1}^{3} l\left(p_{i}, \mathcal{F}\right) E_{i}\right] \cdot \sigma_{*}^{-1}(S),
$$

because the $\overline{r_{i j}}$ do not intersect $\sigma_{*}^{-1}(S)=\sigma^{*}(S)-\sum_{i=1}^{3} E_{i}$. Then as asserted in i):

$$
d\left(Q_{*}(\mathcal{F})\right)=-2+2 \cdot(d(\mathcal{F})+2)-\sum_{i=1}^{3} l\left(p_{i}, \mathcal{F}\right) .
$$

For proving ii), with $\overline{r_{j k}}:=f^{-1}\left(p_{i}^{\prime}\right)(i, j, k \in\{1,2,3\}$ are distinct $)$, write:

$$
l\left(p_{i}^{\prime}, Q_{*}(\mathcal{F})\right)=\left[f^{*}\left(N_{Q_{*}(\mathcal{F})}\right)-l\left(p_{i}^{\prime}, Q_{*}(\mathcal{F})\right) \overline{r_{j k}}\right] \cdot \overline{r_{j k}} .
$$

We assert that this intersection (20) can be computed as:

$$
\left[\sigma^{*}\left(N_{\mathcal{F}}\right)-l\left(p_{j}, \mathcal{F}\right) E_{j}-l\left(p_{k}, \mathcal{F}\right) E_{k}\right] \cdot \overline{r_{j k}}
$$

thanks to $E_{i} \cdot \overline{r_{j k}}=0, E_{j} \cdot \overline{r_{j k}}=1, E_{k} \cdot \overline{r_{j k}}=1$ and the isomorphism:

$$
\begin{gathered}
f^{*}\left(N_{Q_{*}(\mathcal{F})}\right)-l\left(p_{i}^{\prime}, Q_{*}(\mathcal{F})\right) \overline{r_{j k}}-l\left(p_{j}^{\prime}, Q_{*}(\mathcal{F})\right) \overline{r_{i k}}-l\left(p_{k}^{\prime}, Q_{*}(\mathcal{F})\right) \overline{r_{i j}}= \\
=\sigma^{*}\left(N_{\mathcal{F}}\right)-l\left(p_{i}, \mathcal{F}\right) E_{i}-l\left(p_{j}, \mathcal{F}\right) E_{j}-l\left(p_{k}, \mathcal{F}\right) E_{k}
\end{gathered}
$$

which express the factorization $\chi=f \circ \sigma^{-1}$. Then from (20) and (21), we get as desired:

$$
l\left(p_{i}^{\prime}, Q_{*}(\mathcal{F})\right)=d(\mathcal{F})+2-l\left(p_{j}, \mathcal{F}\right)-l\left(p_{k}, \mathcal{F}\right) .
$$




\subsubsection{Examples in dimension two}

Modular foliations The Hilbert modular foliations are pairs of singular foliations which appear after compactification and desingularization of the quotient of the bidisc $\Delta \times \Delta$ by groups of arithmetical nature. They have a very special position in the classification of [1], having $\kappa=-\infty$. After the quotient, the involution which sends the horizontal discs to the vertical ones becomes a birational involution transforming one modular foliation into the other. In [13] there is an explicit description of a pair of modular foliations in the projective plane with degrees five and nine, denoted $\mathcal{H}_{5}$ and $\mathcal{H}_{9}$. There is a degree five birational involution $\chi$ with $\mathcal{H}_{9}=\chi_{*}\left(\mathcal{H}_{5}\right)$ and $\chi$ is a composition $\chi=$ $Q_{3} \circ Q_{2} \circ Q_{1}$ of three standard Cremona transformations, with $\operatorname{Ind}\left(Q_{1}\right)=\left\{a_{1}, a_{2}, a_{3}\right\}$, $\operatorname{Ind}\left(Q_{2}\right)=\left\{b_{1}, b_{2}, b_{3}\right\}, \operatorname{Ind}\left(Q_{3}\right)=Q_{2}\left(\operatorname{Ind}\left(Q_{1}\right)\right)$, for $a_{i} \neq b_{j}$ (more about $\chi$ in the next Example). The foliations $\mathcal{H}_{6}=Q_{1 *}\left(\mathcal{H}_{5}\right)$ and $\mathcal{H}_{8}=\left(Q_{2} \circ Q_{1}\right)_{*}\left(\mathcal{H}_{5}\right)$ have degree six and eight, resp. . It is not asserted in that paper that 5 is the birational degree of $\mathcal{H}_{5}$, but we know that this is true, thanks to Corollary 1 . In fact, all singular points of $\mathcal{H}_{5}$ are either reduced or radial, that is, $l\left(p, \mathcal{H}_{5}\right) \leq 2<\frac{d\left(\mathcal{H}_{5}\right)+2}{3}$. Also we remark that $\mathcal{H}_{6}$ has a point with $l\left(p, \mathcal{H}_{6}\right)=3>\frac{d\left(\mathcal{H}_{6}\right)+2}{3}$; that $\mathcal{H}_{8}$ has a point with $l\left(p, \mathcal{H}_{8}\right)=4>\frac{d\left(\mathcal{H}_{8}\right)+2}{3}$ and that $\mathcal{H}_{9}$ has points with $l\left(p, \mathcal{H}_{9}\right)=4>\frac{d\left(\mathcal{H}_{9}\right)+2}{3}$.

Also in [13] there is a pair of modular foliations $\mathcal{H}_{2}$ and $\mathcal{H}_{3}$, of degrees two and three resp obtained from $\mathcal{H}_{5}$ and $\mathcal{H}_{9}$ by taking quotient with their symmetry group. $\mathcal{H}_{2}$ is not birationally equivalent to a linear foliation or a degree zero foliation (this can be proved directly by considering the leaves of such foliations or as consequence of the birational classification of [1]). Unhappily this fact is not a consequence of Corollary 1 , because $\mathcal{H}_{2}$ has an infinitely near point with $l(p)=2>\frac{d\left(\mathcal{H}_{2}\right)+2}{3}$. This shows that the condition of Corollary 1 is just a sufficient condition, not a necessary one. Also $\mathcal{H}_{3}$ has an infinitely near point with $l(p)=2>\frac{d\left(\mathcal{H}_{3}\right)+2}{3}$ and $\mathcal{H}_{3}$ is equivalent to $\mathcal{H}_{2}$ by a birational involution.

Pencil contained in a homaloidal net It is known that the general Geiser involution (denoted $\chi_{8}$ is given by a homaloidal system $\mathcal{W}_{8}$ of octics $C_{8}$ having triple points at seven general points $q_{i}$. It is known that the Cremona involution $\chi=Q_{3} \circ Q_{2} \circ Q_{1}$ in the previous Example is a degenerate Geiser involution, whose homaloidal system has a fixed part of degree three. So $\chi_{5}:=\chi$ is associated to a net of quintics $C_{5}$ with double points at six points $p_{j}$ in general position. Both exemplify the classical Noether inequalities, which asserts that $\nu\left(q_{i}, C_{8}\right)>\frac{8}{3}$ and $\nu\left(p_{i}, C_{5}\right)>\frac{5}{3}$ for some $i, j$. Suppose now that we fix one extra point $p_{7}$ and consider the pencil $\mathcal{F} \subset \mathcal{W}_{5}$ of quintics passing doubly by $p_{1}, \ldots p_{6}$ and simply by $p_{7}$. Darboux' formula gives $d(\mathcal{F})=8$. We can obtain $\mathcal{F}$ from a pencil of lines $\mathcal{F}_{1}$ applying three times Proposition 1 and the previous factorization $\chi=Q_{3} \circ Q_{2} \circ Q_{1}$, we get: $d\left(\left(Q_{1}\right)_{*}\left(\mathcal{F}_{1}\right)\right)=2$ and $l\left(a_{i},\left(Q_{1}\right)_{*}\left(\mathcal{F}_{1}\right)\right)=2$, $d\left(\left(Q_{2} \circ Q_{1}\right)_{*}\left(\mathcal{F}_{1}\right)\right)=6$ and $l\left(b_{i},\left(Q_{2} \circ Q_{1}\right)_{*}\left(\mathcal{F}_{1}\right)\right)=4, d\left(\left(Q_{3} \circ Q_{2} \circ Q_{1}\right)_{*}\left(\mathcal{F}_{1}\right)\right)=8$ and $l\left(Q_{2}\left(a_{i}\right),\left(Q_{3} \circ Q_{2} \circ Q_{1}\right) *\left(\mathcal{F}_{1}\right)\right)=4$, for $i=1,2,3$.

The Halphen pencils These are pencils $\mathcal{F}_{l \geq 2}$ generated by a singular elliptic curve of degree $3(l-1)$, having 9 points with $\nu(C, p)=l-1$, and by a cubic $C_{3}$ taken with multiplicity $l-1$. They were considered in [14] as foliations with $\kappa=1(\forall l \geq 2)$. We consider in this example generic Halphen pencils, in the sense that $(l-1) C_{3}$ is the unique non-reduced element in the pencil, the nine base-points belong the plane and extra singularities are of Morse type $d(x y)=0$. As foliations, $d\left(\mathcal{F}_{l}\right)=3 l-2$, by 
Darboux formula. The singularities at the base-points have $l\left(p_{i}, \mathcal{F}_{l}\right)=l, \forall i=1, \ldots, 9$ : in fact, representing locally $(l-1) C_{3}$ as $x^{l-1}=0$, we see that the algebraic multiplicity of the holomorphic 1-forms

$$
x^{2(l-1)} \cdot d\left(\frac{\prod_{j=1}^{l-1}\left(y-c_{i} x\right)}{x^{l-1}}\right)
$$

representing $\mathcal{F}_{l}$ at the base points is $m\left(p_{i}, \mathcal{F}_{l}\right)=l-1$. Their blowing ups produce non-invariant exceptional divisors, thanks to the supposition that there are 9 distinct base-points in the plane. As remarked in Section 2.1.1, $l\left(p_{i}, \mathcal{F}_{l}\right)=(l-1)+1=l$. Therefore any $p \in \operatorname{sing}\left(\mathcal{F}_{l}\right)$ has $l\left(p, \mathcal{F}_{l}\right) \leq l=\frac{3 l-2+2}{3}$ and Corollary 1 asserts that $3 l-2$ is the birational degree of $\mathcal{F}_{l}$.

Now consider a standard quadratic transformation $Q$ acting in a Halphen pencil $\mathcal{F}_{l}$, with $\left\{q_{1}, q_{2}, q_{3}\right\}=\operatorname{Ind}(Q)$. If at least one of the $q_{i}$ is not chosen at a base-point of $\mathcal{F}_{l}$ then

$$
d\left(Q_{*}\left(\mathcal{F}_{l}\right)\right)=2(3 l-2)+2-\sum_{j=1}^{3} l\left(q_{j}, \mathcal{F}_{l}\right)>6 l-2-3 l=3 l-2,
$$

by Proposition 1; so the degree is increased. But if all $q_{j}$ are chosen among the basepoints, then $d\left(Q_{*}\left(\mathcal{F}_{l}\right)\right)=2(3 l-2)+2-3 l$, that is, the degree is preserved. The same Proposition gives in this case that the contractions of the strict transforms $\overline{q_{i} q_{k}}$ of the three lines $q_{i} q_{k}$ introduce singularities $r_{k}$ of $Q_{*}\left(\mathcal{F}_{l}\right)$ with:

$$
l\left(r_{k}, Q_{*}\left(\mathcal{F}_{l}\right)\right)=(3 l-2)+2-2 l=l .
$$

Examples from [10] We find there a 1-parameter family of degree 4 foliations $\mathcal{F}_{\lambda}$, with $\lambda \in \mathbf{C} P^{1}$, for which $\kappa=0$. We can assert that the birational degree of such examples is 4 , thanks to the fact that the singularities of $\mathcal{F}_{\lambda}$ are either reduced or radial, that is, $l\left(p, \mathcal{F}_{\lambda}\right) \leq 2=\frac{d\left(\mathcal{F}_{\lambda}\right)+2}{3}$. By other side, it is remarked in that paper that $\mathcal{F}_{\lambda}=\Pi^{*}\left(\mathcal{G}_{\lambda}\right)$ where $\mathcal{G}_{\lambda}$ are foliations of the plane with degree 3 and $\Pi(x, y)=(x+y, x y)$. Of course $\Pi$ is not birational. Such $\mathcal{G}_{\lambda}$ has a singular point with $l\left(p, \mathcal{G}_{\lambda}\right)=2>\frac{3+2}{3}$ and indeed the author uses a standard quadratic transformation $Q$ in order to obtain $Q_{*}\left(\mathcal{G}_{\lambda}\right)$ with degree 2.

Examples from [15] Let us compute the birational degree of the pencil $\mathcal{F}_{k}(\forall k>3)$ in $\mathbf{C} P^{2}$ generated by $C_{1}:=\left\{x_{0}^{k}-x_{1}^{k}=0\right\}$ and $C_{2}:=\left\{x_{1}^{k}-x_{2}^{k}=0\right\}$ (such pencils appear in [15] and have $\kappa=1)$. The pencil contains $C_{3}:=C_{1}+C_{2}:=\left\{x_{0}^{k}-x_{2}^{k}=0\right\}$. By Darboux formula (19), $d\left(\mathcal{F}_{k}\right)=2 \cdot k-2$, because there are no multiple components. By other side, the algebraic multiplicity of the 1-forms $d\left(x^{k}-y^{k}\right)=k x^{k-1} d x-k y^{k-1} d y$ which induce the foliation near the singular points $\left\{p_{1}, p_{2}, p_{3}\right\}$ of $C_{1}, C_{2}$ and $C_{3}$ is $m\left(p, \mathcal{F}_{k}\right)=$ $k-1$ and so $l\left(p_{i}, \mathcal{F}_{k}\right)=k-1>\frac{2 k}{3}$. The standard quadratic transformation $Q$ with $\operatorname{Ind}(Q)=\left\{p_{1}, p_{2}, p_{3}\right\}$ produces $Q_{*}\left(\mathcal{F}_{k}\right)=\mathcal{G}_{k}$ of degree $k+1$. In fact, Proposition 1 gives $d\left(Q_{*}\left(\mathcal{F}_{k}\right)\right)=2 \cdot(2 k-2)+2-3 \cdot(k-1)$ and

$$
l\left(q_{i}, Q_{*}(\mathcal{F})\right)=d(\mathcal{F})+2-l\left(p_{j}, \mathcal{F}\right)-l\left(p_{s}, \mathcal{F}\right)=2, \quad i, j, s \in\{1,2,3\} .
$$

Since the fundamental lines $\left\{x_{0}=0\right\},\left\{x_{1}=0\right\},\left\{x_{2}=0\right\}$ of $Q$ are not $\mathcal{F}$-invariant, the singular points $q_{i}$ of $Q_{*}(\mathcal{F})$ introduced by their contractions are radial points, locally given as $x d y-y d x+$ h.o.t $=0$. Since $k+1<2 k-2$ for $k>3$, we have obtained a reduction of degree of the foliation and thanks to Corollary 1 this degree $k+1$ cannot be birationally decreased. 
Pencils of $3 k$-tics invariant by standard Cremona transformation We give now a pencil of $3 k$-tics $\mathcal{F}_{k}(\forall k \geq 1)$ where each curve is invariant by the standard Cremona transformation and where $l\left(p_{i}, \mathcal{F}_{k}\right)=2 k=\frac{d\left(\mathcal{F}_{k}\right)+2}{3}$ (these assertions can be checked directly or with the software Maple):

$t x y z\left[x^{2(k-1)} y^{k-1}+x^{2(k-1)} z^{k-1}+y^{2(k-1)} x^{k-1}+y^{2(k-1)} z^{k-1}+z^{2(k-1)} x^{k-1}+\right.$ $\left.z^{2(k-1)} y^{k-1}\right]+x^{2 k}\left(y^{k}+z^{k}\right)+y^{2 k}\left(x^{k}+z^{k}\right)+z^{2 k}\left(x^{k}+y^{k}\right)=0$.

Coverings of pencils in the plane Take $f(x: y: z)=\left(x^{2}: y^{2}: z^{2}\right)$. By composing with $f$, we obtain from the next pencil of cubics $\mathcal{F}_{3}$, the pencil of sextics $\mathcal{F}_{6}$ :

$\mathcal{F}_{3}: x^{2}(y+z)+y^{2}(x+z)+z^{2}(x+y)+t x y z=0, \mathcal{F}_{6}: x^{4}\left(y^{2}+z^{2}\right)+y^{4}\left(x^{2}+z^{2}\right)+$ $z^{4}\left(x^{2}+y^{2}\right)+t y^{2} z^{2} x^{2}=0$.

Each sextic is invariant by the standard Cremona map in the plane. We compute at the indetermination points $p_{i}: l\left(p_{i}, \mathcal{F}_{6}\right)=3$. Darboux formula (19) asserts $d\left(\mathcal{F}_{6}\right)=$ $2(6)-2-3=7$, since for the parameter $t=\infty$ the curve $V: y^{2} z^{2} x^{2}=0$ is a cubic with multiplicity 2 . So $l\left(p_{i}, \mathcal{F}_{6}\right)=\frac{d\left(\mathcal{F}_{6}\right)+2}{3}$.

\subsection{Higher dimension}

\subsubsection{Cubo-cubic Cremona transformation}

The cubo-cubic Cremona transformation $\chi$ is a birational involution of $\mathbf{C} P^{3}$, given by the four $3 \times 3$ minors of a $3 \times 4$ matrix of linear forms. The indetermination set $\operatorname{Ind}(\chi)$ is a smooth (connected) twisted sextic $C_{6}$ of genus 3 . Lines and planes are sent respectively to twisted cubics intersecting $\operatorname{Ind}\left(\chi^{-1}\right)$ in eight variable points and cubic surfaces passing simply by $\operatorname{Ind}\left(\chi^{-1}\right)$. Its factorization $\chi=f \circ \sigma^{-1}$ is very simple (and characterizes the cubo-cubic transformation among the Cremona maps of $\mathbf{C} P^{3}[8]$ ): $\sigma: M \rightarrow \mathbf{C} P^{3}$ is just one blowing up along $\operatorname{Ind}(\chi)$ and $f$ is the contraction of a surface $\bar{S} \subset M$ to a curve $C_{6}^{\prime}=\operatorname{Ind}\left(\chi^{-1}\right)$ isomorphic to $C_{6}$. Where $\bar{S}$ is the strict transform of an octic $S$ passing triply along $C_{6}$. This surface $S$ is the scroll of trisecant lines of $C_{6}$. Denoting $E=\sigma^{-1}(\operatorname{Ind}(\chi))$, then $\chi_{*}(\operatorname{Ind}(\chi))=f_{*}(E)$ is again an octic surface passing triply by $C_{6}^{\prime}$, the scroll of trisecants of $C_{6}^{\prime}$. Keeping these notations:

Proposition 2 Let $\mathcal{F}$ be a foliation of $\mathbf{C} P^{3}$. Let $\chi$ denote the cubo-cubic Cremona transformation of $\mathbf{C} P^{3}$. Then

i): $d\left(\chi_{*}(\mathcal{F})\right)=3 \cdot d(\mathcal{F})+4-8 \cdot l(\operatorname{Ind}(\chi), \mathcal{F})$.

ii): $l\left(\operatorname{Ind}\left(\chi^{-1}\right), \chi_{*}(\mathcal{F})\right)=d(\mathcal{F})+2-3 \cdot l(\operatorname{Ind}(\chi), \mathcal{F})$.

Proof

For i), start with $d\left(\chi_{*}(\mathcal{F})\right)=-2+N_{\chi_{*}(\mathcal{F})} \cdot L$ as in $(6)$, where the line $L$ is the birational image of a twisted cubic $C_{3}$ intersecting $\operatorname{Ind}(\chi)$ in eight variable points. Let $\overline{C_{3}}$ and $\bar{S}$ denote the strict transforms by $\sigma$ of $C_{3}$ and of the scroll $S$. Remark that $C_{3} \cdot S=24$ is given by 8 points of $\operatorname{ind}(\chi)$ counted with multiplicity 3 . Then $\overline{C_{3}} \cdot \bar{S}=0$. This means that the subsequent contraction of $\bar{S}$ by $f$ does not affect $\overline{C_{3}}$; we get:

$$
N_{\chi_{*}(\mathcal{F})} \cdot L=N_{\sigma_{*}^{-1}(\mathcal{F})} \cdot \overline{C_{3}}=(d(\mathcal{F})+2) \cdot 3-8 \cdot l(\operatorname{Ind}(\chi), \mathcal{F}) .
$$

For ii), denote $\overline{l_{\text {tris }}}$ and $\bar{S}$ the strict transforms by $\sigma$ of the trisecant lines $l_{\text {tris }} \subset S$ and of $S$. Since $\bar{S}=f^{-1}\left(C_{6}^{\prime}\right)$, we get $\bar{S} \cdot \overline{l_{\text {tris }}}=-1$ and as asserted:

$$
l\left(\operatorname{Ind}\left(\chi^{-1}\right), \chi_{*}(\mathcal{F})\right)=\left[f^{*}\left(N_{\chi_{*}(\mathcal{F})}\right)-l\left(\operatorname{Ind}\left(\chi^{-1}\right), \chi_{*}(\mathcal{F})\right) \cdot \bar{S}\right] \cdot \overline{l_{\text {tris }}}=
$$




$$
=\sigma_{*}^{-1}\left(N_{\mathcal{F}}\right) \cdot \overline{l_{\text {tris }}}=d(\mathcal{F})+2-3 \cdot l(\operatorname{Ind}(\chi), \mathcal{F}) .
$$

We remark that, in particular, if $d(\mathcal{F})$ or the multiplicity $l(\operatorname{Ind}(\chi), \mathcal{F})$ is preserved by $\chi$, then Proposition 2 gives:

$$
d(\mathcal{F})+2=4 \cdot l(\operatorname{Ind}(\chi), \mathcal{F})
$$

which agrees with the conclusion of Theorem 1-ii).

\subsubsection{Classical flops of the standard cubic transformation}

The standard Cremona transformation of $\mathbf{C} P^{3}$ (easily generalized to higher dimensions) is given by $C(x: y: z: w)=(y z w: x z w: x y w: x y z)$ and has as indetermination set the six edges of the fundamental tetrahedron $\{x y z w=0\}$. Twisted cubics through the four vertices are sent to lines and cubic surfaces with double points at the vertices are sent to planes. There is a factorization

$$
C=f \circ \sigma^{-1}=f_{I I} \circ f_{I} \circ\left(\sigma_{I} \circ \sigma_{I I}\right)^{-1},
$$

where $\sigma_{I}$ is the blowing up of the four vertices $v_{i}$ of the tetrahedron and $\sigma_{I I}$ is the blowing up along the strict transforms $\overline{r_{i j}}$ by $\sigma_{I}$ of the 6 edges $r_{i j}:=v_{i} v_{j}$. The six exceptional divisors $E_{i j}=\sigma_{I I}^{-1}\left(\overline{r_{i j}}\right)$ are then collapsed by $f_{I}$ to rational curves ${\overline{r_{i j}}}^{\prime}$. The last step is $f_{I I}$, which contracts to points $v_{i}^{\prime}$ the strict transforms by $f_{I} \circ \sigma^{-1}$ of the four planes of the fundamental tetrahedron. The classical flop (cf. [19]) is:

$$
f_{I} \circ \sigma_{I I}^{-1}
$$

whose effect is to introduce one of the rulings of $E_{i j}=\mathbf{C} P^{1} \times \mathbf{C} P^{1}$ and to collapse the other ruling, sending the curves $\overline{r_{i j}}$ to ${\overline{r_{i j}}}^{\prime}$. Therefore the vertices of the tetrahedron $v_{i} \subset \operatorname{Ind}(C)$ have divisorial birational image by $C$, namely, the fundamental planes of another tetrahedron, although the edges $r_{i j} \subset \operatorname{Ind}(C)$ of the tetrahedron are just flopped. With these notations:

Proposition 3 Let $\mathcal{F}$ be a foliation of $\mathbf{C} P^{3}$. Let $C$ denote the standard cubic Cremona transformation, let $v_{1}, \ldots, v_{4}$ be the vertices of the fundamental tetrahedron and $v_{1}^{\prime}, \ldots, v_{4}^{\prime}$ denote the vertices of the new tetrahedron composed by the birational images of $v_{1}, \ldots, v_{4}$. Then:

i): $d\left(C_{*}(\mathcal{F})\right)=3 \cdot d(\mathcal{F})+4-\sum_{i=1}^{4} l\left(v_{i}, \mathcal{F}\right)$ and

ii): $l\left(v_{i}^{\prime}, C_{*}(\mathcal{F})\right)=2 \cdot d(\mathcal{F})+4-\sum_{j \neq i} l\left(v_{j}, \mathcal{F}\right), j \in\{1,2,3,4\}$.

\section{Proof}

For i), start with $d\left(C_{*}(\mathcal{F})\right)=-2+N_{C_{*}(\mathcal{F})} \cdot L$, for a line $L$ which is the strict transform by $C$ of a twisted cubic $T$ through $v_{1}, \ldots, v_{4}$. We assert that $N_{C_{*}(\mathcal{F})} \cdot L$ is computed as

$$
N_{C_{*}(\mathcal{F})} \cdot L=\left[\sigma_{I}^{*}(\mathcal{F})-\sum_{i=1}^{4} l\left(v_{i}, \mathcal{F}\right) E_{i}\right] \cdot\left[\sigma_{I}^{*}(T)-\sum_{i=1}^{4} e_{i}\right],
$$

where $E_{i}:=\sigma^{-1}\left(v_{i}\right)$ and $e_{i} \subset E_{i}$ is a line, with $E_{i} \cdot e_{i}=-1$. In other words, we assert that it suffices to consider just the effect of $\sigma_{I}$. The reason for this is that the rational curve $\sigma_{I}^{*}(T)-\sum_{i=1}^{4} e_{i}$ neither intersects the strict transforms by $\sigma_{I}$ of the edges of 
the tetrahedron (which will be flopped by $f_{I} \circ \sigma_{I I}^{-1}$ ) nor intersects the strict transforms of the fundamental planes (which will be collapsed by $f_{I I}$ ). Then from $(22)$ we get as desired:

$$
d\left(C_{*}(\mathcal{F})\right)=-2+3 \cdot(d(\mathcal{F})+2)-\sum_{i=1}^{4} l\left(v_{i}, \mathcal{F}\right) .
$$

For ii), denote $E_{i}^{\prime}=f_{I I}^{-1}\left(v_{i}^{\prime}\right)$, where $E_{i}^{\prime}$ are the strict transforms of the fundamental planes generated by $v_{j}, v_{k}, v_{s}$, for $i \neq j, k, s \in\{1,2,3,4\}$. We can write:

$$
l\left(v_{i}^{\prime}, C_{*}(\mathcal{F})\right)=\left[f_{I I}^{*}\left(N_{C_{*}(\mathcal{F})}\right)-l\left(v_{i}^{\prime}, C_{*}(\mathcal{F})\right) E_{i}^{\prime}\right] \cdot e_{i}^{\prime}, \quad e_{i}^{\prime} \subset E_{i}^{\prime}, \quad e_{i}^{\prime} \cdot E_{i}^{\prime}=-1 .
$$

We assert that the intersection (23) can be computed as:

$$
\left[\sigma_{I}^{*}\left(N_{\mathcal{F}}\right)-l\left(v_{i}, \mathcal{F}\right) E_{i}-l\left(v_{j}, \mathcal{F}\right) E_{j}-l\left(v_{k}, \mathcal{F}\right) E_{k}-l\left(v_{s}, \mathcal{F}\right) E_{s}\right] \cdot\left[\sigma_{I}^{*}(S)-e_{j}-e_{k}-e_{s}\right],
$$

where $S$ are conics passing by $v_{j}, v_{k}, v_{s}$, contained in the fundamental plane generated by them, and where $E_{i} \cdot e_{j}=0, i \neq j, E_{j} \cdot e_{j}=-1$. In fact, $e_{i}^{\prime}=\sigma_{I}^{*}(S)-e_{j}-e_{k}-e_{s}$ and the reason for forgetting the effect of the flop $f_{I} \circ \sigma_{I I}^{-1}$ in the computation of (23) is that the rational curve $\sigma_{I}^{*}(S)-e_{j}-e_{k}-e_{s}$ does not intersect the (strict transforms) of the edges of the tetrahedron. Therefore from (24) we get, as desired:

$$
l\left(v_{i}^{\prime}, C_{*}(\mathcal{F})\right)=2 \cdot(d(\mathcal{F})+2)-l\left(v_{j}, \mathcal{F}\right)-l\left(v_{k}, \mathcal{F}\right)-l\left(v_{s}, \mathcal{F}\right)
$$

\subsubsection{Examples in higher dimension}

Pencil of Enriques sextics The Enriques surfaces are special surfaces which have singular models in $\mathbf{C} P^{3}$ given by a family of surfaces of degree 6 , whose singular set are the edges of a tetrahedron (passing doubly by the edges and triply by the vertices). There is a sub-family with tetrahedral symmetry. From this sub-family we found the following pencil $\mathcal{F}$, where each curve is invariant by the cubic standard Cremona map: $y z^{2} w^{2} x+y^{2} z w^{2} x+y^{2} z^{2} w x+x^{2} z w^{2} y+x^{2} z^{2} w y+x^{2} y^{2} w z+t\left[x^{2} y^{2} z^{2}+x^{2} y^{2} w^{2}+\right.$ $x^{2} z^{2} w^{2}+y^{2} z^{2} w^{2}+2 y z^{2} w^{2} x+2 y^{2} z w^{2} x+2 y^{2} z^{2} w x+2 x^{2} z w^{2} y+2 x^{2} z^{2} w y+2 x^{2} y^{2} w z+$ $\left.x y z w^{3}+x y z^{3} w+x y^{3} z w+x^{3} y z w\right]=0$.

This is an example for Theorem 1 -ii), since $d(\mathcal{F})=2 \cdot 6-2=10$ and for the vertices $v_{i, j, k}=\left\{x_{i}=x_{j}=x_{k}=0\right\}$ we can compute: $l(v, \mathcal{F})=6=2 \cdot \frac{10+2}{4}$.

Pencil of $4 k$-tic surfaces We have examples of pencils $\mathcal{F}_{k}$ composed by surfaces of degree $4 k(\forall k \geq 1)$, each one invariant by the standard cubic Cremona transformation: $x^{2 k}\left(y^{k} z^{k}+y^{k} w^{k}+z^{k} w^{k}\right)+y^{2 k}\left(x^{k} z^{k}+x^{k} w^{k}+z^{k} w^{k}\right)+z^{2 k}\left(x^{k} y^{k}+x^{k} w^{k}+y^{k} w^{k}\right)+$ $w^{2 k}\left(x^{k} y^{k}+x^{k} z^{k}+y^{k} z^{k}\right)+t \cdot x y z w\left[x^{2(k-1)} y^{2(k-1)}+x^{2(k-1)} z^{2(k-1)}+x^{2(k-1)} w^{2(k-1)}+\right.$ $\left.y^{2(k-1)} z^{2(k-1)}+y^{2(k-1)} w^{2(k-1)}+z^{2(k-1)} w^{2(k-1)}\right]=0$.

At the vertices $v_{i}$ of $\{x y z w=0\}: l\left(v_{i}, \mathcal{F}_{k}\right)=4 k=(3-1) \cdot \frac{d\left(\mathcal{F}_{k}\right)+2}{4}$.

Examples from [17] J.V. Pereira gave me an example of a pencil $\mathcal{F}_{k}$ in $\mathbf{C} P^{3}$ composed by $2 k$-tics $(k \geq 1)$ :

$$
\left(x^{k}-y^{k}\right)\left(z^{k}-w^{k}\right)+t\left(x^{k}-z^{k}\right)\left(y^{k}-w^{k}\right)=0,
$$

where each surface in the pencil is invariant by the standard Cremona transformation. We can compute at the vertices $v$ of the fundamental tetrahedron: $l\left(v, \mathcal{F}_{k}\right)=2 k=$ $2 \cdot \frac{d\left(\mathcal{F}_{k}\right)+2}{4}$. 
Coverings of pencils in the space Composing with $f(x: y: z: w)=\left(x^{2}: y^{2}: z^{2}: w^{2}\right)$ we get, from the next pencil of quartics $\mathcal{F}_{4}$, the following pencil $\mathcal{F}_{8}$ of octics :

$\mathcal{F}_{4}: t x y z w+x^{2}(y z+y w+z w)+y^{2}(x z+x w+z w)+z^{2}(x y+x w+y w)+w^{2}(x y+$ $x z+y z), \quad \mathcal{F}_{8}: t x^{2} y^{2} z^{2} w^{2}+x^{4}\left(y^{2} z^{2}+y^{2} w^{2}+z^{2} w^{2}\right)+y^{4}\left(x^{2} z^{2}+x^{2} w^{2}+z^{2} w^{2}\right)+$ $z^{4}\left(x^{2} y^{2}+x^{2} w^{2}+y^{2} w^{2}\right)+w^{4}\left(x^{2} y^{2}+x^{2} z^{2}+y^{2} z^{2}\right)=0$

where each surface is invariant by the standard Cremona map in the space. For the vertices $v$ :

$$
l\left(v, \mathcal{F}_{8}\right)=6=2 \cdot \frac{2(8)-2-4+2}{4}=2 \cdot \frac{d\left(\mathcal{F}_{8}\right)+2}{4},
$$

because Darboux formula $(19)$ gives $d\left(\mathcal{F}_{8}\right)=2(8)-2-4$, since for the parameter $t=\infty$ the surface $V: x^{2} y^{2} z^{2} w^{2}=0$ is a quartic with multiplicity $\mu=2$.

Examples in $\mathbf{C} P^{4}$ and $\mathbf{C} P^{N}$ We give here an example with quintics in $\mathbf{C} P^{4}$, which can be easily generalized to $5 k$-tics in $\mathbf{C} P^{4}$ or in general of $(N+1) \cdot k$-tics in $\mathbf{C} P^{N}$. A pencil $\mathcal{F}_{5}$ of quintic hypersurfaces where each hypersurface is invariant by the standard (quartic) Cremona transformation is:

$$
x^{2}(s w z+w y s+y z s+y z w)+y^{2}(z w s+x w s+x z s+x z w)+z^{2}(y w s+x w s+x y s+
$$
$x y w)+w^{2}(y z s+x z s+x y s+x y z)+s^{2}(y z w+x z w+x y w+x y z)+t x y z w s=0$.

Here we compute for the vertices of the tetrahedron $\{x y z w s=0\}: l\left(v, \mathcal{F}_{5}\right)=6=$ $(4-1) \cdot \frac{d\left(\mathcal{F}_{5}\right)+2}{5}$.

Pencils of quartic surfaces invariant by the cubo-cubic Cremona map Based on [4], let us give an example with $d(\mathcal{F})=6$ and $l(\operatorname{Ind}(\chi), \mathcal{F})=2$, where $\mathcal{F}$ is a pencil of quartic surfaces and each surface is invariant by the cubo-cubic map $\chi$. For this, firstly remark any surface $\pi$ with birational image $\chi_{*}(\pi)$ produces a curve $\pi \cap \chi_{*}(\pi)$ which is $\chi$-invariant: in fact, $\chi$ is an involution. Secondly, for each line $l$ let $\pi_{l, t}$ be a pencil of planes (with parameter $t$ ) containing $l$ and consider its birational image $\chi_{*}\left(\pi_{l, t}\right)$, which is a cubic surface. Consider now the $\chi$-invariant plane cubic $C_{l, t}:=\pi_{l, t} \cap \chi_{*}\left(\pi_{l, t}\right)$. Varying $\pi_{l, t}$ in the pencil of planes we produce a (possibly singular) surface $S_{l}$, with

$$
\left(C_{l, t} \cup \operatorname{Ind}(\chi)\right) \subset S_{l}, \quad \forall t,
$$

passing simply by the curve $\operatorname{Ind}(\chi)$ (cf. [4]). Since the points of $C_{l, t} \cap l \in S_{l}$ depend on $t$ we see that $l \subset S_{l}$. Then, for each $t, S_{l} \cap \pi_{t}=C_{l, t} \cup l$ is a degree four plane curve, so $\operatorname{deg}\left(S_{l}\right)=4$. Being composed by $\chi$-invariant curves $C_{l, t}$, the surfaces $S_{l}$ are $\chi$-invariant. Varying now $l$ we get a linear system of quartics $S_{l}$, from which we take a pencil of quartics denoted $\mathcal{F}$. The degree of $\mathcal{F}$ is $d(\mathcal{F})=2 \cdot 4-2$ and $l(\operatorname{Ind}(\chi), \mathcal{F})=2$ since the quartics in the pencil $\mathcal{F}$ can be taken transverse to each other along $\operatorname{Ind}(\chi)$ (locally $\mathcal{F}$ is given by $x d y-y d x+$ h.o.t $=0$ along $\operatorname{Ind}(\chi)$ ). Since $\chi_{*}(\mathcal{F})=\mathcal{F}$ and $\operatorname{Ind}(\chi)$ has codimension 2 , we get: $l(\operatorname{Ind}(\chi), \mathcal{F})=2=(2-1) \cdot \frac{d(\mathcal{F})+2}{4}$.

Pencils of surfaces whose degree can be decreased There is a pencil $\mathcal{F}$ of sextic surfaces passing doubly by the curve $\operatorname{Ind}(\chi)$ of the cubo-cubic transformation and $\mathcal{F}$ has a cubic surface counted twice. Darboux formula gives $d(\mathcal{F})=2 \cdot 6-3-2=7$. Taking in account the non reduced element we can compute $l(\operatorname{Ind}(\chi), \mathcal{F})=3$, analogously to what happens with Halphen pencils in Section 4.1.2. So $l(\mathcal{F}, \operatorname{Ind}(\chi))>\frac{7+2}{4}$ and $\mathcal{F}^{\prime}=\chi_{*}(\mathcal{F})$ with $d\left(\mathcal{F}^{\prime}\right)=1$ is a pencil of quadrics not passing by $\operatorname{Ind}\left(\chi^{-1}\right)$, having a plane counted twice. By other side, if $\mathcal{G}$ is a pencil of sextics passing doubly by the curve $\operatorname{Ind}(\chi)$ and free of non-reduced elements, then $d(\mathcal{G})=2 \cdot 6-2=10, l(\operatorname{Ind}(\chi), \mathcal{G})=4$ 
and $l(\operatorname{Ind}(\chi), \mathcal{G})=4>\frac{10+2}{4}$. The transformed pencil $\chi_{*}(\mathcal{G})$ is composed by quadrics and has $d\left(\mathcal{G}^{\prime}\right)=2$.

At last, in [5] there are quintic surfaces in $\mathbf{C} P^{3}$ having four triple points along the vertices of the fundamental tetrahedron, for which $l(v, \mathcal{F})=6$ (compare with Example 4.2.3). Such pencil of quintics, considered as foliation, has $d(\mathcal{F})=8$ and so $6>5=2 \cdot \frac{d(\mathcal{F})+2}{4}$. The transformed pencil by the standard cubic map is a pencil of cubics $\mathcal{F}^{\prime}$ with degree $d\left(\mathcal{F}^{\prime}\right)=4$.

Acknowledgements Firstly I thank Professor Marcos Sebastiani, who asked many years ago the question discussed by Corollary 1 , which motivated this work. I also thank the attention of Francesco Russo and of Jorge V. Pereira. At last, I am grateful to Ivan Pan.

\section{References}

1. M. Brunella, Birational geometry of foliations, First Latin American Congress of Mathematicians, IMPA, 2000 (www.impa.br/Publicacoes/Monografias/index.html).

2. M. Brunella, Minimal models of foliated algebraic surfaces, Bull. Soc. Math. France, 127 (2): 289-305, 1999.

3. S. Cantat and C. Favre, Symétries birationnelles des surfaces feuilletées, J. Reine Angew. Math, 561, 199-235 (2003); corrigendum ibid 582, 229-231 (2005).

4. W. L. EdGe, Notes on a net of quadric surfaces. I. The Cremona transformation, Proc. Lond. Math. Soc., II. Ser. 43, 302-315 (1937).

5. S. Endrass, U. Persson and J. Stevens, Surfaces with triple points, J. Algebr. Geom. 12, No.2, 367-404 (2003).

6. E. Esteves And S. Kleiman, Bounds on leaves of one-dimensional foliations, Bull. Braz. Math. Soc. (N.S.) 34 (2003), no. 1, 145-169.

7. V.A. Iskovskikh and Yu. Prokhorov, Fano varieties, Algebraic Varieties V, 1-247, Encyclopaedia Math. Sci., 47, Springer Verlag (1999).

8. S. KATZ, The cubo-cubic transformation of $\mathbf{C} P^{3}$ is very special, Math. Z. 195 (1987), no. $2,255-257$.

9. J. Kollár, K. E. Smith And A. Corti, Rational and nearly rational varieties, Cambridge University Press, 2004.

10. A. Lins Neto, Some examples for Poincaré and Painlevé problems, Ann. Scient. Éc. Norm. Sup., 35, 231-266 (2002).

11. M. MCQUillan, Non-commutative Mori theory, preprint IHES, 2001.

12. L. G. Mendes, Kodaira dimension of holomorphic singular foliations, Bol. Soc. Brasil. Mat. (N.S.) 31: 127-143, 2000.

13. L.G. Mendes and J.V. Pereira, Hilbert modular foliations in the projective plane, Comment. Math. Helv. 80, 243-291, 2005.

14. L.G. Mendes And P. SAD, On dicritical foliations and Halphen pencils, Ann. Sc. Norm. Super. Pisa, Cl. Sci. (5) 1, No.1, 93-109 (2002).

15. J.V. PEREIRA AND P. SAD, On the rigidity of certain holomorphic fibrations, in preparation.

16. J.V. Pereira AND P. F. SANChez, Transformation groups of holomorphic foliations, Communications in Analysis and Geometry, 10, n.5, 1115-1123 (2002).

17. J. V. Pereira and S. Yuzvinsky, Completely reducible hypersurfaces in a pencil, preprint, IMPA, 2007.

18. H. Poincaré, Sur l'integration algébrique des équations différentielles dupremier ordre et du premier degré, Rend.Circ. Mat. Palermo 5 (1891), 161-191 and 11 (1897), 193239.

19. M. ReID, Twenty five years of 3-folds. An old person's view in Corti, Alessio (ed.) et al., Explicit birational geometry of 3-folds, Cambridge University Press. Lond. Math. Soc. Lect. Note Ser. 281, 313-343 (2000). 